\title{
Bioactive Compounds from Germinated Brown Rice Protect Cardiomyocytes Against Simulated Ischemic/Reperfusion Injury by Ameliorating Mitochondrial Dysfunction
}

This article was published in the following Dove Press journal:

Drug Design, Development and Therapy

\author{
Kanokwan Demeekul $\mathbb{D}^{\prime}$ \\ Wichit Suthammarak ${ }^{2}$ \\ Soontaree Petchdee (D) ${ }^{3}$ \\ 'Graduate School, Program of Bio- \\ Veterinary Science, Kasetsart University, \\ Kamphaeng Saen, Nakorn Pathom, \\ Thailand; ${ }^{2}$ Department of Biochemistry, \\ Faculty of Medicine Siriraj Hospital, \\ Mahidol University, Bangkok, Thailand; \\ ${ }^{3}$ Department of Large Animal and \\ Wildlife Clinical Sciences, Faculty of \\ Veterinary Medicine, Kasetsart \\ University, Kamphaeng Saen Campus, \\ Nakorn Pathom, Thailand
}

Purpose: Ischemic/reperfusion (I/R) injury is the principal mechanism during Ischemic Heart Disease (IHD). The key modulator of $\mathrm{I} / \mathrm{R}$ injury is dysregulation of mitochondria function. Germinated Brown Rice (GBR) has been recommended as a bio-functional food and has clarified the potential properties in several effects. However, the effect of GBR mediated cardioprotective properties, focusing on mitochondrial function's role, remains unexplored. Thus, this study aims to investigate the cardioprotective effects of GBR pretreatment against simulated $\mathrm{I} / \mathrm{R}$ injury.

Methods: H9c2 cardiomyocytes were incubated with GBR at a five $\eta \mathrm{g} / \mathrm{mL}$ concentration for 24 hours and simulated I/R (sI/R) for 40 minutes. Cell viability and cell apoptosis were assessed by 7-AAD staining and Annexin V/PI staining, respectively. The mitochondrial membrane potential was determined by JC-1 staining and mitochondrial respiration represented by oxygen consumption rate (OCR) using Seahorse Flux analyzer.

Results: The results revealed that the administration of GBR before sI/R significantly decreased the percentage of cell death and total cell apoptosis in H9c2 during stimulation of ischemic/reperfusion. Besides, pretreatment of cardiomyocytes with GBR remarkably stabilized mitochondrial membrane potential and improved impaired mitochondrial respiration in simulated-H9c2 injury.

Conclusion: The present research is the first study to report the effective cardioprotection of GBR. Pretreatment of GBR potentially protects $\mathrm{H} 9 \mathrm{c} 2$ cardiomyocytes against sI/R injury through mitochondrial function. The underlying therapeutic activities are possibly associated with its bio-functional compounds. However, the underlying mechanism on the cardioprotective effects of GBR needs further studies.

Keywords: cardioprotection, germinated brown rice, GBR, H9c2 cardiomyocyte, ischemia reperfusion injury, mitochondria function

\section{Introduction}

Ischemic heart disease (IHD) is considered the single largest cause of death worldwide and is estimated to increase morbidity and mortality in the next coming decade. ${ }^{1}$ Principally, an insufficient blood supply causes IHD to heart tissues due to occlusion of the arterial blood flow. Inadequate tissue perfusion leads to ischemia-induced tissue damage and cell death. ${ }^{2}$ The presence of attenuated blood flow in the heart response to myocardial ischemia can lead to myocardial infarction (MI). ${ }^{3,4}$ Not only ischemia that
Correspondence: Soontaree Petchdee Department of Large Animal and Wildlife Clinical Sciences, Faculty of Veterinary Medicine, Kasetsart University,

Kamphaeng Saen, Nakorn Pathom, 73I40, Thailand

Tel +6634351901-3

Fax +663435I405

Email fvetstr@ku.ac.th 
occurred on myocardium damage but also reperfusion. Reperfusion is a rapid restoration of arterial blood flow to the ischemic myocardium. ${ }^{5}$ The adverse results of ischemic and reperfusion processes are called ischemia/reperfusion (I/ R) injury. ${ }^{6}$ The critical mechanism of $\mathrm{I} / \mathrm{R}$ injury reveals that ischemia derogates ATPase-dependent ion-exchange channels and interrupts cell volume regulation, which leads to lysis of organelle and plasma membranes and disrupts enzymatic activity. $^{7,8}$ Also, reperfusion enhances reactive oxygen and nitrogen species production by impairing mitochondria function and triggers a series of pro-inflammatory cytokines release, endoplasmic reticulum stress, and tissue development damage. The well-known end-effector of I/R-induced cell injury and death is the opening of mitochondrial permeability transition pores (MPTP). ${ }^{2}$ Many recent studies emphasize the role of mitochondrial dysfunction, which is associated with I/ R injury. ${ }^{6-14}$ Therefore, maintaining impaired cardiac mitochondrial function could be a useful therapeutic target of $I / R$ injury.

In recent years, attractive alternative medication, such as herbal therapies and dietary supplements, has remarkably increased because of their variety of curative properties. ${ }^{15}$ Much of the evidence recommended the therapeutic effects of bio-functional components in various daily diets, such as antioxidant, ${ }^{16,17}$ antimicrobial property, ${ }^{18}$ cardioprotective effects, and diminishing the risk factors of cardiovascular diseases, such as hypertension, hyperinsulinemia, dyslipidemia, or arteriosclerosis in vitro and animal models. ${ }^{19,20}$ Several current studies demonstrated that many Asian countries tend to have a lower risk of cardiovascular diseases than European and American countries regarding their consumer behavior and cultivated areas. ${ }^{21}$ One such plant food is rice, mostly cultured in Asia for consumption worldwide. Brown rice is important rice that has lots of essential nutritional contents and bioactive compounds. However, a previous study reported that bioactive components gained more during the germination process. $^{22}$ Germinated brown rice (GBR) contains many bio-functional components, such as ferulic acid, $\gamma$-oryzanol, and gamma-aminobutyric acid (GABA). ${ }^{23-25}$ Several physiological effects of GBR have been demonstrated as an antihyperlipidemic by increasing cholesterol catabolism, antihypertension and exhibited to lower risk of chronic diseases, including cancer, diabetes, cardiovascular diseases, and Alzheimer's disease. ${ }^{26}$ Hence, it may be possible that GBR could be biologically active in human health due to its benefits. By the way, the underlying mechanism of GBR, especially cardiovascular diseases focusing on mitochondria function, is not revealed. Additionally, there is still a lack of information about the potential mechanism of cardioprotective properties of GBR. Therefore, this study aims to investigate the cardioprotective effect of GBR during myocardial I/R injury.

\section{Materials and Methods \\ Chemicals and Reagents}

Dulbecco's modified Eagle's medium (DMEM), fetal bovine serum, and trypsin-EDTA purchased from Gibco ${ }^{\circledR}$; Life Technologies Inc. FITC Annexin V Apoptosis Detection Kit I obtained from BD Pharmingen, USA. MitoScreen (JC-1) and Via-probe (7-AAD staining) purchased from BD Bioscience, USA. XF Cell Mito Stress Test kit purchased from Seahorse Bioscience, USA.

\section{The Extraction of Bioactive Compound of GBR}

Brown jasmine rice (Oryza sativa L.) has obtained from Sakonnakorn province, Thailand. Germinated brown rice seeds were dried at $50^{\circ} \mathrm{C}$ overnight. Germinated seeds were ground and stored at $-20^{\circ} \mathrm{C}$ and used for subsequent analyses. Germinated brown rice (GBR) was extracted with $80 \%$ ethanol (ratio 1:2) at room temperature for 72 hours and filtrated. The maceration process was repeated two times. Each filtrate was pooled and evaporated under reduced pressure to dryness. The extracts were further freeze-dried to produce GBR crude extracts. The crude extract was subsequently partitioned by hexane, dichloromethane, and ethyl acetate to give crude hexane, dichloromethane, ethyl acetate, and water extracts, respectively.

\section{Cell Culture}

The rat cardiac myoblast cell line $(\mathrm{H} 9 \mathrm{c} 2)$ was originated from embryonic BD1X rat cardiac tissue and purchased from American Type Cell Culture (ATCC-CRL1446). $\mathrm{H} 9 \mathrm{c} 2$ cell line was routinely cultured as a monolayer in Dulbecco's Modified Eagle's Medium (DMEM) (Gibco ${ }^{\circledR}$ ) with $10 \%$ fetal bovine serum (FBS), amphotericin, penicillin $\mathrm{G}$, and streptomycin under $\mathrm{pH} 7.4$ at $37^{\circ} \mathrm{C}$ in humidified air containing $5 \% \mathrm{CO}_{2}$. The cell density of $70 \%$ confluence in passage number 5-10 was used for all experiments. The culture medium was changed with a fresh warm medium every 3 days.

\section{Cell Treatment}

Cells were pretreated with various times (10-120 minutes) and concentrations of germinated brown rice crude 
extraction $(0-100 \mu \mathrm{M})$ to achieve appropriate time and concentration for the further study, and then the partitioned by hexane, dichloromethane, and ethyl acetate and water extraction were evaluated for cellular protection during myocardial ischemia (Data not shown).

\section{Simulated Ischemia in $\mathrm{H} 9 \mathrm{c} 2$ Cardiomyocyte Cell}

The ischemic injury in vitro model was established as previously described. ${ }^{27}$ Briefly, we provided sodium dithionite to induce the simulated ischemic condition. The H9c2 cardiomyocytes were incubated in $2 \mathrm{~mL}$ of ischemic buffer (137 mM NaCl, $3.8 \mathrm{mM} \mathrm{KCl}, 0.49 \mathrm{mM}$ $\mathrm{MgCl} 2,1.8 \mathrm{mM} \mathrm{CaCl} 2$ and $4.0 \mathrm{mM}$ HEPES), followed by $20 \mathrm{mM} 2$ deoxyglucose, $30 \%$ sodium lactate and $1 \mathrm{mM}$ sodium dithionite at $\mathrm{pH} 6.3$, in order to induce sI/R in 24 well plate. Ischemic induction was performed in the period of time for 40 minutes as previously described. ${ }^{28}$ After ischemic induction, the ischemic buffer was replaced and the cell was incubated with DMEM with $10 \%$ fetal bovine serum (FBS) for all experimental assessments.

\section{Determination of Cell Death by 7-Aminoactinomycin D (7-AAD) Viability Staining}

7-AAD cell viability staining used in this study is based on specific binding to double-stranded DNA with high affinity. The H9c2 cardiomyocyte cells were plated in a 24-well plate at a density of $2 \times 10^{5}$ cells per well and maintained in $2 \mathrm{~mL}$ DMEM with $10 \%$ fetal bovine serum (FBS) for 24 hours. They were then pretreated with or without a concentration of 5 $\eta \mathrm{g} / \mathrm{mL}$ GBR and further incubated for 24 hours. After 40 minutes of $s \mathrm{I} / \mathrm{R}$ induction, the cells were collected at a concentration of $1 \times 10^{5}$ cells $/ \mathrm{mL}$ and then stained with 20 $\mu \mathrm{L}$ of 7-AAD in $100 \mu \mathrm{L}$ of PBS for 20 minutes in the dark at room temperature. The percentage of 7-AAD-stained cells was optimized by Accuri C6 flow cytometer (BD Bioscience, USA) for the viability 7-AAD staining and presented as a bar graph.

\section{Determination of Cell Apoptosis by Annexin V/Propidium lodine (PI) Assay}

Cellular apoptosis was evaluated by Annexin V/PI assay. $\mathrm{H} 9 \mathrm{c} 2$ cells were seeded at a density of $2 \times 10^{5}$ cells per well in a $24-w e l l$ plate. The medium was discarded, followed by pretreatment with or without concentrations of $5 \mathrm{ng} / \mathrm{mL}$ GBR for 24 hours. After 72 hours of incubation, sI/R induction was performed as the previous experiment. Briefly, cultured medium in each well was collected, and the cells were washed with PBS, followed by trypsinization. Approximately $1 \times 10^{5}$ cells/ $\mathrm{mL}$ in each condition was collected and centrifuged. Then, the cell pellets were mixed with $100 \mu \mathrm{L}$ of $1 \mathrm{X}$ binding buffer and $3 \mu \mathrm{L}$ of Annexin V solution and incubated in a dark room for 10 minutes. Then, this cell was incubated with $2 \mu \mathrm{L}$ of PI solution for 5 minutes. Finally, cell apoptosis was determined by flow cytometry using Accuri C6 flow cytometer (BD Bioscience, USA). The percentage of total cell apoptosis was presented as a bar graph.

\section{Determination of Mitochondria Membrane Potential Alteration $(\Delta \psi \mathrm{m})$}

Mitochondria membrane potential alteration was detected by JC-1 staining. The qualitative data of mitochondrial membrane potential were assessed by fluorescence microscopy (Operetta CLSTM high content analysis system, PerkinElmer, USA). H9c2 cardiomyocyte cells were cultured at a density of $1 \times 10^{4}$ cells per well in a 96-well plate in the cultured medium. After 24 hours, cells were pretreated with or without concentrations of $5 \mathrm{ng} / \mathrm{mL}$ GBR for 24 hours and exposed to sI/R for 40 minutes. 5\% DMSO was used as a positive control of the experiment. Then, cultured media was removed and cells were washed with PBS. After that, the cells were incubated with JC1 working solution at $37^{\circ} \mathrm{C}$ with a $5 \% \mathrm{CO}_{2}$ incubator for 1 hour in dark. Finally, the mitochondrial membrane potential changes were immediately analyzed by fluorescence microscopy using the Columbus image data storage and analysis system.

For further analysis of mitochondria membrane potential alteration, the quantitative measurement was performed by flow cytometry. The density of H9c2 cardiomyocyte cells at $2 \times 10^{5}$ cells/well were seeded into 24-well plate in the cultured medium. The cells were harvested by trypsinization after treatment as the previous experiment. On the day of the experiment, cultured media was collected and H9c2 cells were washed and centrifuged. After that, approximately $1 \times 10^{5}$ cells $/ \mathrm{mL}$ in each condition were collected and then were incubated with JC-1 working solution according to the manufacturer's protocol. Finally, the quantitative analysis of red/ green fluorescence intensity ratio was evaluated by using 
Table I Bioactive Compounds of GBR

\begin{tabular}{|l|c|c|}
\hline Phenolic Contents & Total Flavonoid Content & GABA \\
\hline (mg GAE/I00g DW) & (mg CE/100g DW) & (mg GABA/I00g DW) \\
\hline I55.95-146.7I & $44.59-38.73$ & $13.35-12.65$ \\
\hline
\end{tabular}

Abbreviation: DW, dried weight.

Accuri C6 flow cytometer (BD Bioscience, USA) by measuring in the FL1 (530 nm) and FL2 (585 nm) channels for fluorescence compensation controls analysis.

\section{Determination of Mitochondrial} Respiration

A mitochondrial respiratory study was conducted by measuring the oxygen consumption rate (OCR) that reflexed all key parameters of mitochondrial respiration. The H9c2 cardiomyocyte cells at a density of $1.5 \times 10^{4}$ cells per assay were cultured in the XFp cell culture miniplate. Prior to the day of assay, cells were pretreated with concentrations of either $5 \mathrm{ng} /$ $\mathrm{mL} \mathrm{GBR}$ for 24 hours or $\mathrm{s} / \mathrm{R}$ induction for 40 minutes. On the day of assay, the cultured medium was discarded with XF assay medium-modified with $1 \mathrm{mM}$ pyruvate, $2 \mathrm{mM}$ glutamine, and $10 \mathrm{mM}$ glucose and then incubated at $37^{\circ} \mathrm{C}$ without $\mathrm{CO}_{2}$ for $1 \mathrm{~h}$. Then, the cells were serially exposed three times to $5 \mu \mathrm{M}$ of oligomycin, $2 \mu \mathrm{M}$ of FCCP and $0.5 \mu \mathrm{M}$ of rotenone plus antimycin A, respectively, using the XF Cell Mito Stress Test kit (Seahorse Bioscience). The parameters of OCR were measured according to the manufacturer's protocol by using the Seahorse XFp Extracellular Flux analyzer and software (Seahorse Bioscience, USA). All mitochondrial respiratory parameters were calculated and presented as a bar graph.

\section{Statistical Analysis}

All data are expressed as the mean \pm standard error of mean (SEM) at least three independent experiments. Comparisons between different groups were performed by one-way analysis of variance (ANOVA) followed by Tukey's multiple comparison test using GraphPad Prism 8 software. Statistical significance was set at $\mathrm{P}<0.05$.

\section{Results}

\section{Bioactive Compounds of GBR}

The active principles compounds of germinated brown rice such as phenolic contents, total flavonoid content and GABA are shown in Table 1. The total phenolic, flavanoid and GABA contents were 155.95-146.71 mg GAE, 44.59-38.73 mg CE and $13.35-12.65 \mathrm{mg}$ GABA, respectively.

\section{The Protective Property of GBR Against sl/R in H9c2 Cardiomyocyte}

We first determined the protective effect of GBR on sI/ $\mathrm{R}$ induced-H9c2 cardiomyocyte cell death. After pretreated with or without $5 \mathrm{ng} / \mathrm{mL}$ of GBR for 72 hours and followed by sI/R induction, H9c2 cardiomyocyte cells were treated with DMSO as a control. Then, cell viability was determined by 7-AAD staining in flow cytometric plot (Figure 1A) and percentage of 7-AADstained cells (Figure 1B). The results demonstrated that GBR pretreatment remarkably decreased cell death when compared to sI/R condition $(8.31 \%$ vs $11.99 \%$, respectively) (Figure 1A), while induction of sI/R significantly increased the percentage of dead cells when compared to control (11.99\% vs $9.51 \%$, respectively). A summary of the findings on the percentage of cell death is shown in Figure 1B. This result suggested that GBR pretreatment reduced sI/R-induced cardiomyocyte cell death.

\section{Anti-Apoptotic Effect of GBR on H9c2 Cardiomyocyte After sl/R Induction for 72 Hours}

To further determine the anti-apoptotic effect of GBR, H9c2 cardiomyocyte cells were pretreated with or without $5 \mathrm{\eta g} / \mathrm{mL}$ of GBR for 72 hours and followed by sI/R induction for 40 minutes. Then, total cell apoptosis was assessed by Annexin V/PI staining and displayed on the dot plot (Figure 2A). The results showed that pretreatment with GBR significantly reduced H9c2 cardiomyocyte cells apoptosis $(9.57 \%)$ as same as observed in GBR co-treatment with sI/R group $(8.36 \%)$, whereas a higher percent of total cell apoptosis was observed in the sI/R group, compared to control $(15.47 \%$ vs $9.94 \%$, respectively) (Figure 2B). This finding suggested 
A
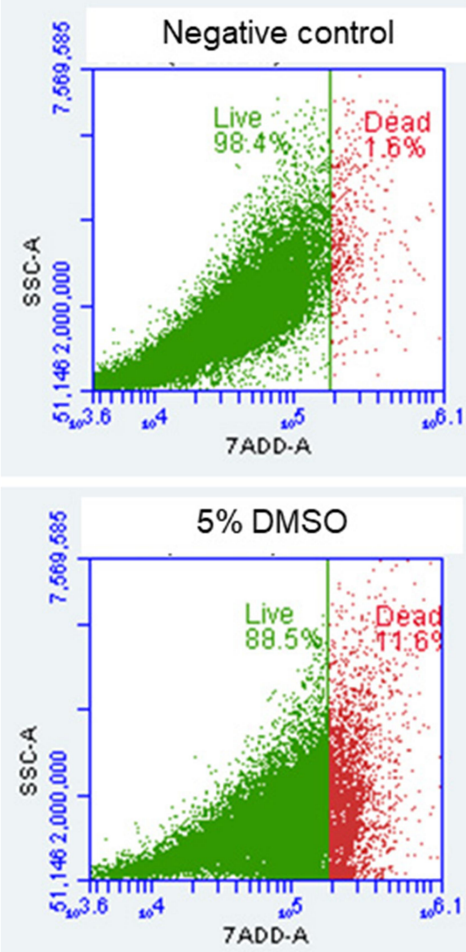
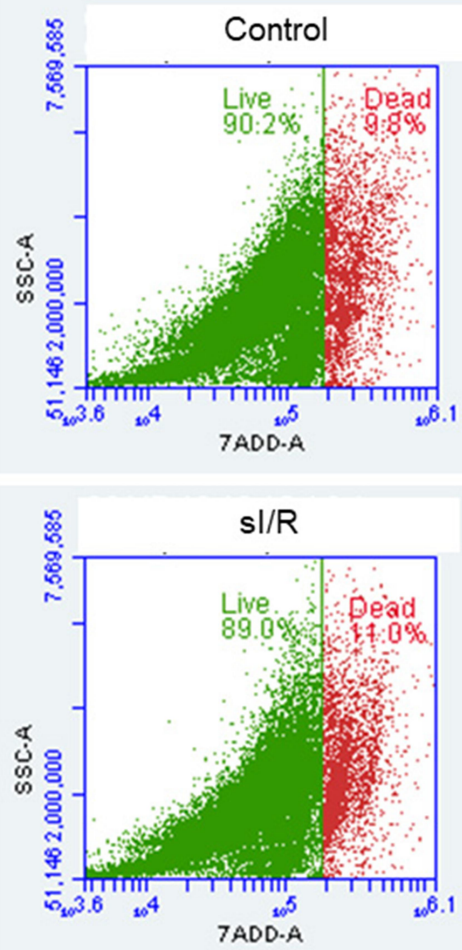
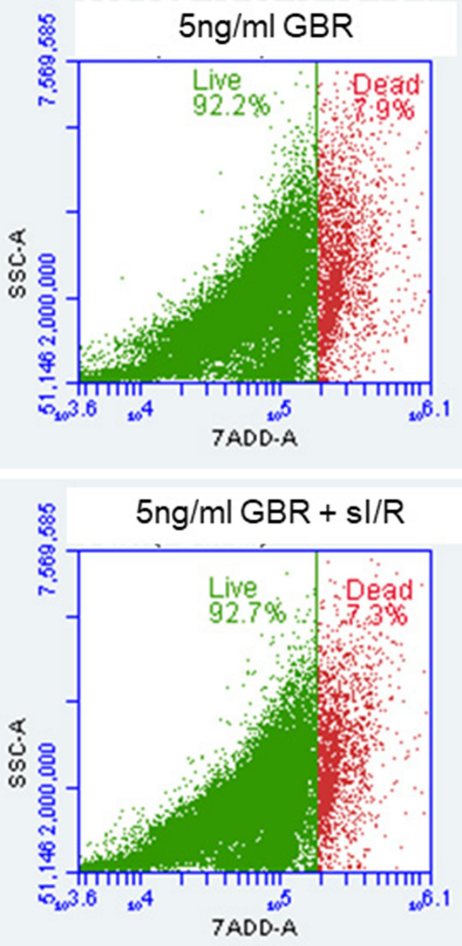

B

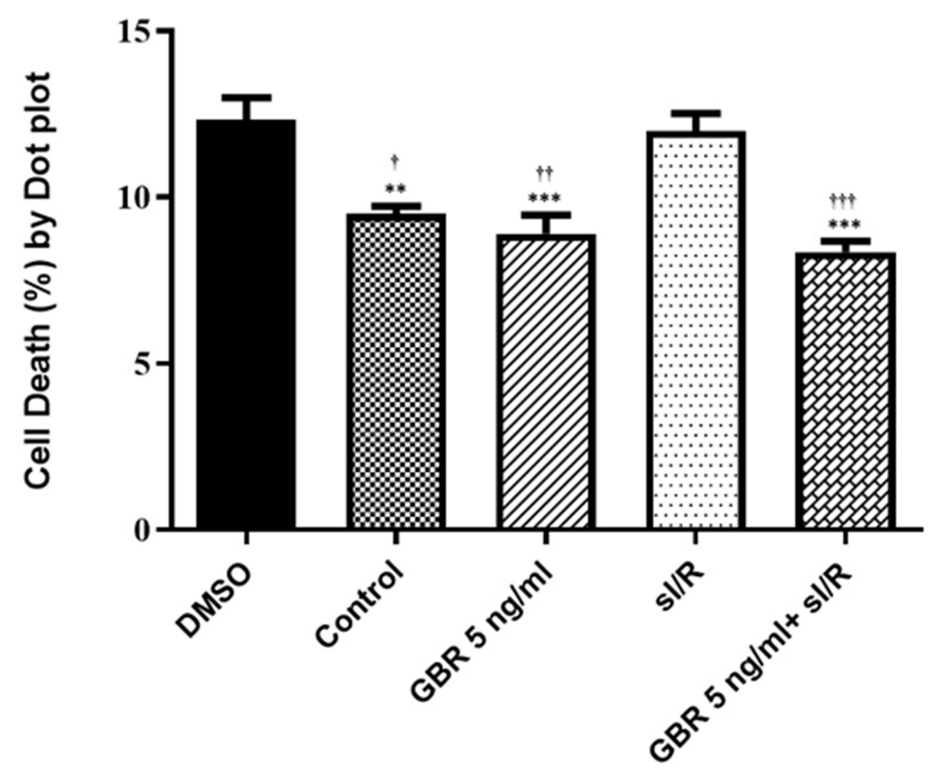

Figure I The protective property of GBR against sl/R in H9c2 cardiomyocyte. H9c2 cardiomyocytes were pretreated with or without 5 ng/mL GBR for 12 hours and then exposed to sl/R for 40 minutes. The determination of cell viability was evaluated by flow cytometry. (A) Flow cytometer plot for 7-AAD staining cell. (B) The percentage of 7-AAD-stained cells was analyzed by flow cytometry. All data was analyzed by One-way ANOVA with Tukey's multiple comparison test. The mean \pm SEM of six individual experiments is shown. ${ }^{* *} \mathrm{P}<0.0 \mathrm{I},{ }^{* * *} \mathrm{P}<0.00 \mathrm{I}$ significant different from DMSO; ${ }^{\dagger} \mathrm{P}<0.05,{ }^{\dagger \dagger} \mathrm{P}<0.0 \mathrm{I},{ }^{\dagger+\dagger} \mathrm{P}<0.00 \mathrm{I}$ significant different from sl/R. 

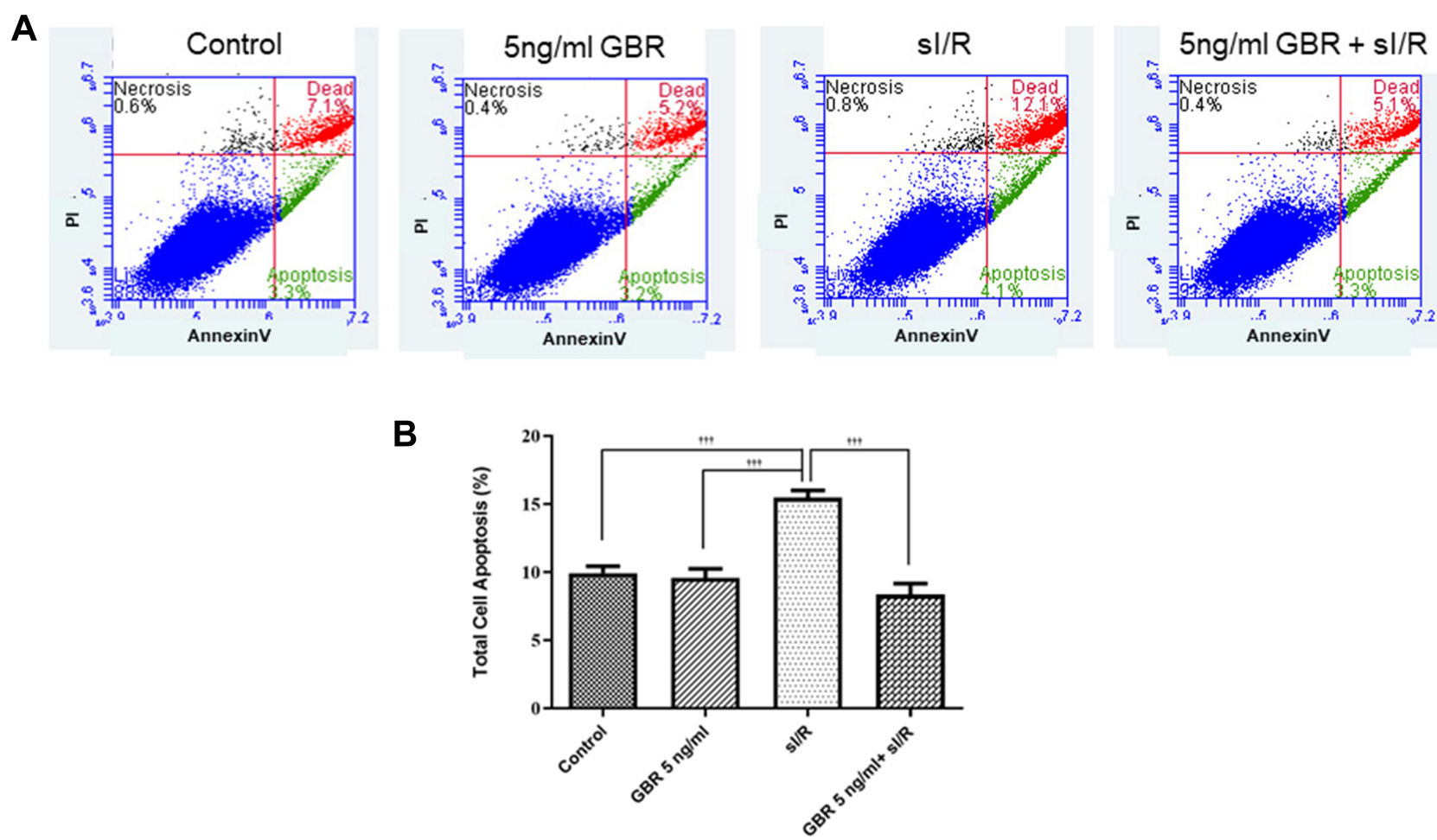

Figure 2 Anti-apoptotic effect of GBR on H9c2 cells during sl/R induction. The determination of anti-apoptotic effect of GBR on sl/R-induced H9c2 cell apoptosis was performed by Annexin V/PI staining. H9c2 cells were pretreated with or without $5 \mathrm{ng} / \mathrm{mL}$ of GBR for 72 hours and followed by sl/R induction. Then, total cell apoptosis was assessed. (A) Flow cytometer dot plot of Annexin V/PI staining. (B) The percentage of total cell apoptosis in H9c2 cardiomyocytes was analyzed by flow cytometry. All data

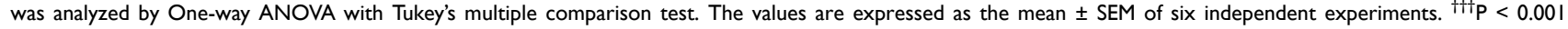
significant different from $\mathrm{s} / \mathrm{R}$.

that GBR treatment exhibited the cardioprotective effect on $\mathrm{S} / \mathrm{R}$ induced cardiomyocyte apoptosis.

\section{Determination of the Effect of GBR on Mitochondrial Transmembrane Potential $(\Delta \Psi \mathrm{m})$ Alteration against sl/R in $\mathrm{H} 9 \mathrm{c} 2$ cardiomyocyte}

This study further determined the cardioprotective effect of GBR on mitochondria function by JC-1 staining. At the end of the experiment, the qualitative analysis of mitochondria membrane potential alteration was observed under fluorescence microscopy as shown in Figure 3A. In addition, the percentage of JC-1 fluorescence intensity in each group was calculated relative to control (Figure $3 \mathrm{~B}$, whereas the quantitative JC-1 analysis was performed by flow cytometry (Figure $3 \mathrm{C}$ and $\mathrm{D}$ ). These results demonstrated that pretreatment with GBR declined the mitochondrial membrane depolarization which was represented by the high intensity of red fluorescence. On the other hand, sI/R treated cells clearly gained the mitochondrial membrane potential disruption as indicated by the decreased red fluorescence, however, increased green fluorescence (Figure 3A and C). Thus, the fluorescence intensity of GBR pretreatment was stronger than sI/ R-treated cells. Furthermore, quantitation of JC-1 staining was further confirmed by flow cytometry. These quantitative results were shown and accomplished by calculating the ratio of the red channel (living cell) with the green channel (non-living cell) (Figure 3C and D). Results showed that cardiomyocyte JC-1-stained cells were conspicuously shifted from red to green fluorescence in $\mathrm{SI} / \mathrm{R}$ condition compared with that in control cells, indicating mitochondria membrane potential dissipation (Figure 3C). Interestingly, this pattern was reversed by pretreatment of GBR which was dramatically increased the red/green ratio compared with those in $\mathrm{sI} / \mathrm{R}$ alone treated cells (Figure 3D). All of these results indicated that GBR pretreatment improved the mitochondrial membrane potential disruption on $\mathrm{H} 9 \mathrm{c} 2$ cell-induced sI/R. 
A
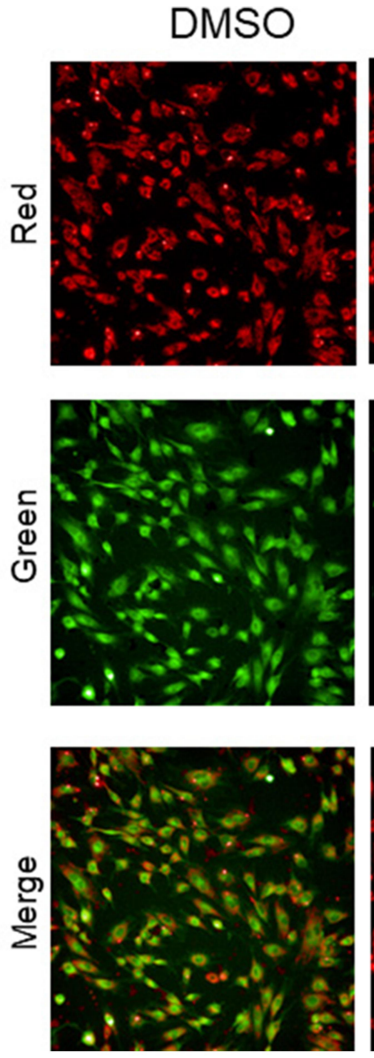
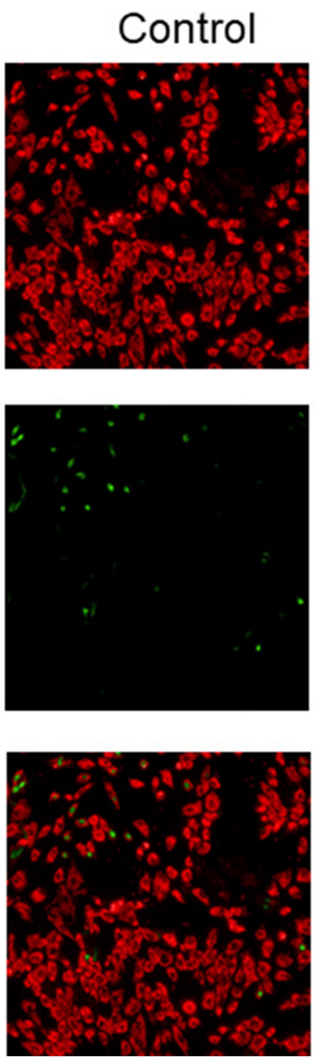
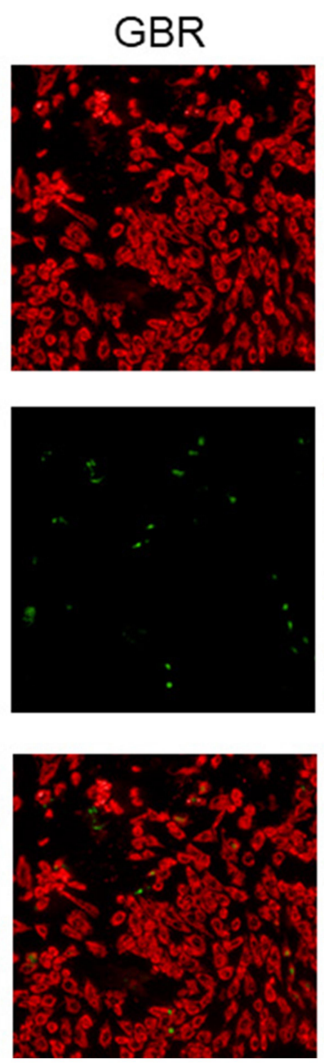
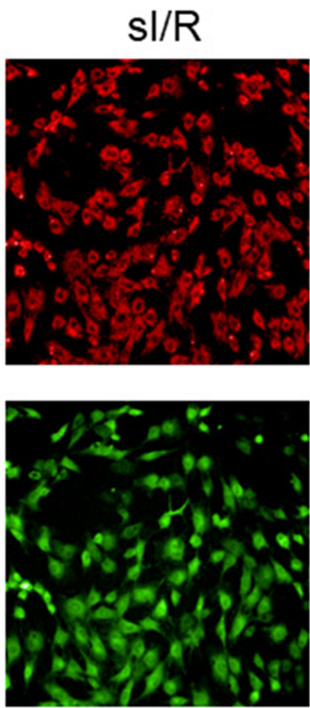

\section{GBR+sl/R}
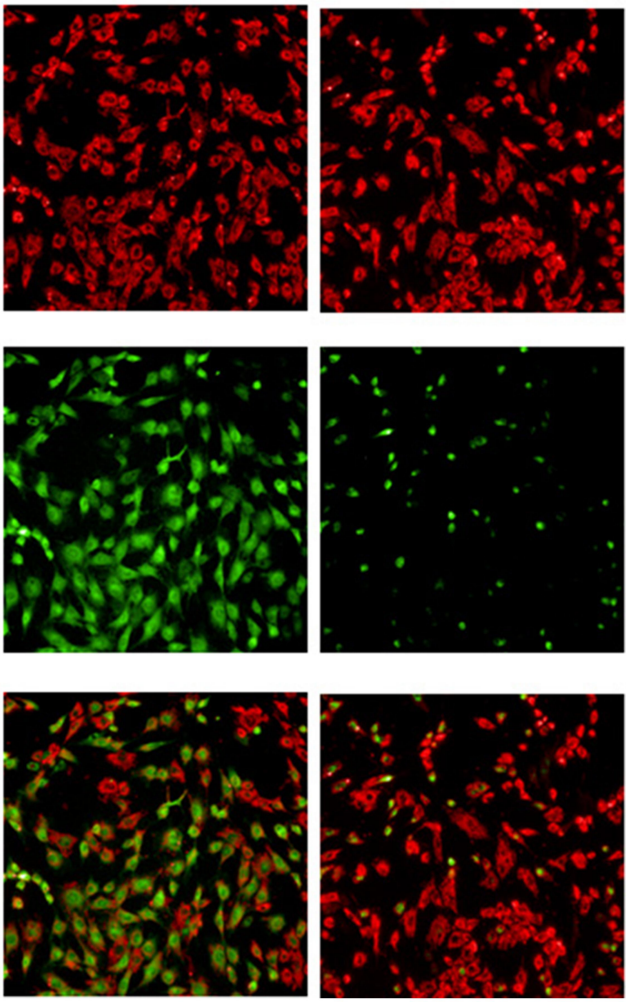

B

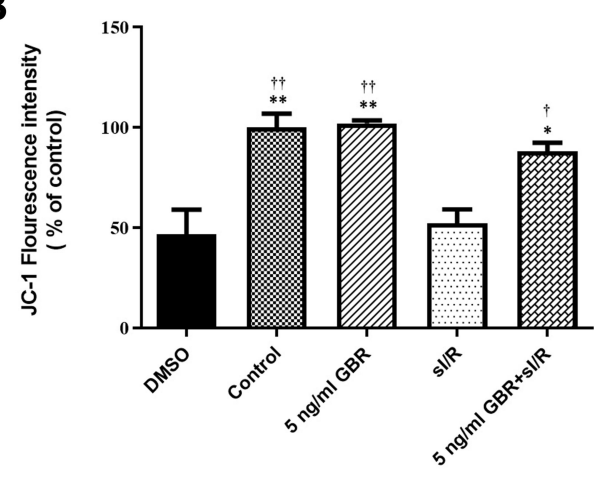

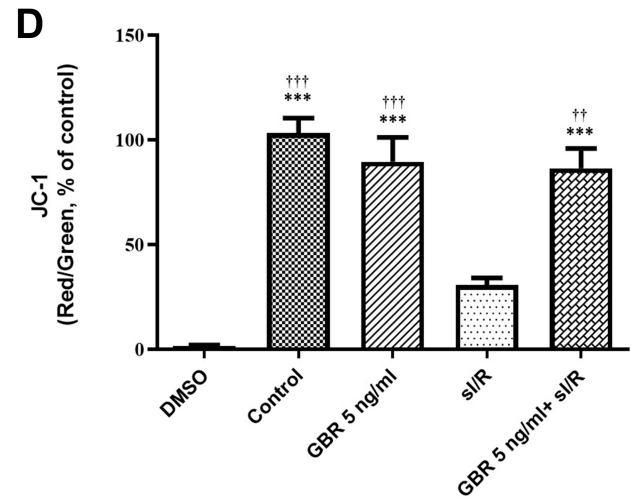

C

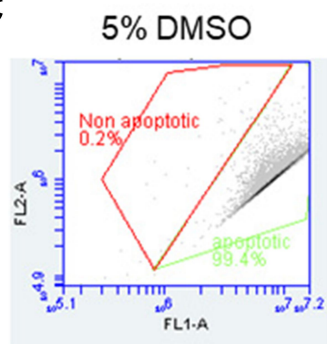

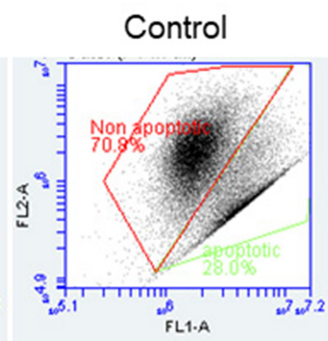
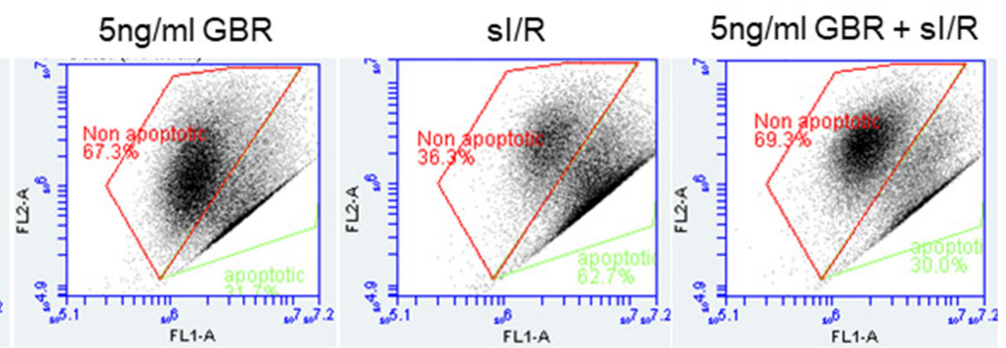

Figure 3 Protective effect of GBR on mitochondrial transmembrane potential disruption against sl/R induction. H9c2 cardiomyocytes were incubated with or without 5 ng/ $\mathrm{mL} \mathrm{GBR}$ for 12 hours and then exposed to sl/R for 40 minutes. Then, mitochondria membrane potential was investigated by JC-I staining. (A) Qualitative analysis of mitochondria membrane potential was assessed by fluorescence microscopy with JC-I staining. (B) JC-I Fluorescence intensity was calculated using Columbus image data storage and analysis system. (C) Scatter diagram of JC-I staining by flow cytometer. (D) Quantitative analysis of red/green ratio. All values were analyzed by one-way ANOVA with Tukey's multiple comparison test. The representative images were collected in three separated images per experiment from at least three independent

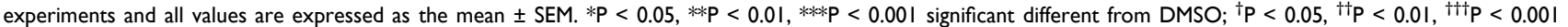
significant different from sl/R. 

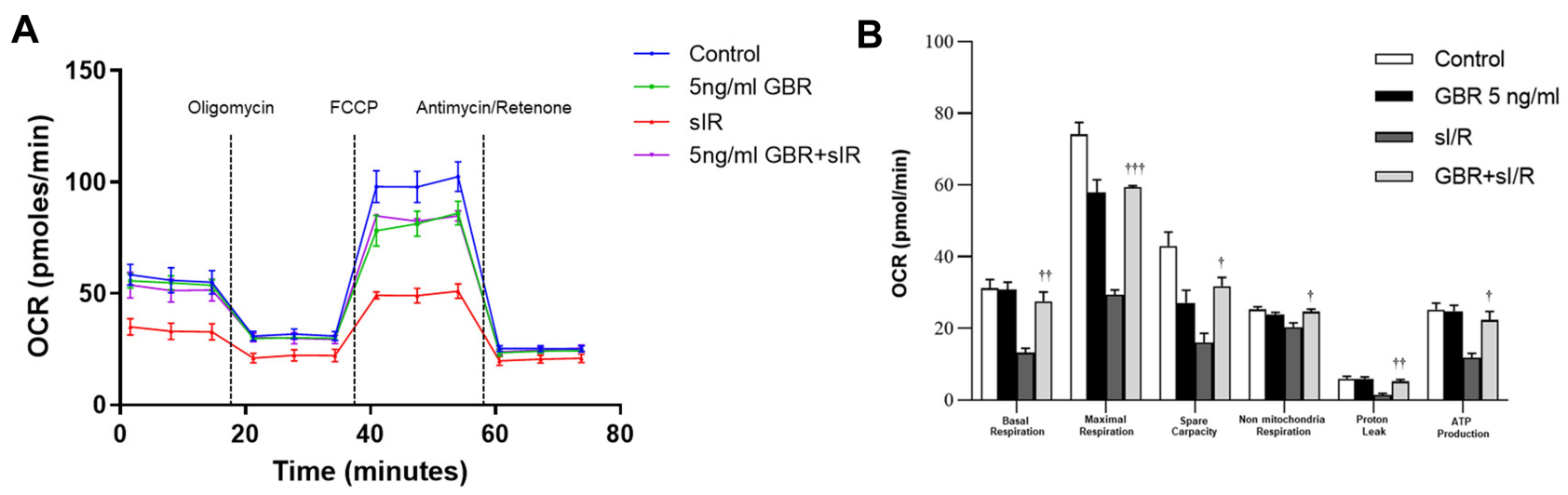

Figure 4 Effect of GBR on mitochondrial respiration against sl/R induction. (A) Time period for measurement of oxygen consumption rate (OCR) was shown using Seahorse XFp analyzer, followed by the serial injection of oligomycin $(5 \mu \mathrm{M})$, FCCP $(2 \mu \mathrm{M})$ and rotenone $(0.5 \mu \mathrm{M})$ plus antimycin $(0.5 \mu \mathrm{M})$. (B) Representative mitochondrial respiration parameters, including basal respiration, maximal respiration, spare capacity, non-mitochondrial respiration, proton leak, and ATP production were evaluated. All values were analyzed by one-way ANOVA with Tukey's multiple comparison test. The values are expressed as the mean \pm SEM of three independent experiments. ${ }^{\dagger} P<0.05$, ${ }^{\dagger \dagger} \mathrm{P}<0.01,{ }^{\dagger \dagger} \mathrm{P}<0.001$ significant different from $\mathrm{sl} / \mathrm{R}$.

\section{Evaluation of Mitochondrial Respiration on GBR Treatment Against sI/R Induction}

The mitochondria respiration was determined through the oxygen consumption rate (OCR) on pretreatment of GBR in sI/R condition. In the study, we used XF Cell Mito Stress Test kit and analyzed by using the Seahorse XFp Extracellular Flux analyzer and software (Seahorse Bioscience, USA). The mitochondria respiration profile is shown in Figure 4A. The representative mitochondrial respiration parameters indicated that GBR pretreatment significantly increased all of the mitochondrial respiration parameters, including basal respiration, maximal respiration, spare respiratory capacity, mitochondrial ATP production, proton leak, and non-mitochondrial respiration to different levels when compared with $\mathrm{sI} / \mathrm{R}$ treated cell. While, the $\mathrm{sI} / \mathrm{R}$ induction dramatically decreased all of these parameters as shown in Figure 4B. Overall, these data suggested that GBR pretreatment preserved the mitochondrial function on sI/R-treated $\mathrm{H} 9 \mathrm{c} 2$ cells.

\section{Discussion}

There are several therapeutic approaches to bio-functional foods against cardiovascular diseases, both in experimental and clinical studies. Germinated Brown Rice (GBR) exerted its protective properties on cardiovascular diseases and related-cardiovascular metabolism dysfunction, including hypertension, hyperlipidemia, and diabetes. Here, this is the first study to represent the cardioprotective effect of GBR on cardiomyocyte-simulated I/R injury. The significant findings in this study indicated that simulation of I/R promoted the percentage of cell death and total cell apoptosis. Additionally, ischemic/reperfusion induction disrupted the mitochondria membrane potential and mitochondria respiration. On the other hand, pretreatment with GBR could significantly reverse all impacts of simulated I/ $R$ injury. Therefore, GBR can offer cardioprotection on $I / R$ injury.

Rice is a primary dietary food and mostly cultivation in Asian countries. Several studies suggested that brown rice contained rich bio-function components, including phenolic contents, gamma-aminobutyric acid (GABA), $\gamma$ oryzanol, ferulic acid, and dietary fibers. ${ }^{29}$ Besides that, brown rice's health beneficial properties mostly contributed to the germination process, mainly throughout metabolic and phytochemicals alteration of GBR. ${ }^{30}$ Our previous study demonstrated several methods, including hexane, dichloromethane, ethyl acetate, and water extracts owing to GBR crude extraction. ${ }^{31}$ Due to that, this study preferred GBR crude extraction from the water extraction method for the whole experimental study.

Moreover, a previous study reported that GBR exhibited protective activity in both the in vitro and animal models with chronic myocardial infarction. ${ }^{28,31}$ The cardioprotective activities of GBR extract were hypothesized by the action of phenolic compounds as major bioactive compounds. $^{28,31}$ Consistent with the present study, we found that GBR mostly contains phenolic contents, total flavonoid content, and GABA, respectively.

GBR treatment results showed to decline in blood pressure in spontaneously hypertensive animal models and humans. ${ }^{32,33}$ Additionally, the administration of GBR 
has been indicated to improve the lipid profiles in obese mice $^{32}$ and decrease the level of hepatoma-induced HDL cholesterol in rats. ${ }^{34}$ Moreover, GBR has been found to suppress hypercholesterolemia via upregulating cholesterol catabolism. ${ }^{35}$ Similar observations in many epidemiological and clinical studies suggested that higher consumption of dietary fiber can affect the lower cardiovascular risk through LDL cholesterol oxidation. ${ }^{36-38}$ This evidence documented that GBR exerts its nutritional and health-promoting benefit properties due to its several biofunction components.

To further investigate the cardioprotective effect of GBR at the molecular level, we first determined the percentage of cell death and apoptosis. Our results indicated that GBR pretreatment significantly protected cardiomyocyte cell death and apoptosis from sI/R induction. Several studies recommended that bioactive components play an essential role in these effects. ${ }^{26}$ Besides, treatment of GBR revealed the therapeutic effect on cancer cell proliferation and apoptosis. ${ }^{39}$

Mitochondria function plays a crucial role in the mechanism of myocardial I/R injury. Moreover, several cellular processes, such as endoplasmic reticulum stress, intracellular calcium overload, and activated apoptotic pathway, are involved in myocardial $\mathrm{I} / \mathrm{R}$ injury. ${ }^{7,9}$ Therefore, mitochondrial function preservation mostly maintained the mitochondrial membrane potential and mitochondria respiration, which may benefit from myocardial ischemic/reperfusion injury. ${ }^{40}$ In this study, our results demonstrated that GBR pretreatment significantly preserved the mitochondrial membrane potential disruption and enhanced mitochondria respiration in simulated ischemic cardiomyocytes. Therefore, it is essential to note that the bio-function components could explain the protective effect of GBR in sI/R injury. Despite the advantage in this study provided by a mitochondrial association of heart cell protection, it cannot mimic the pathophysiological changes during ischemic reperfusion injury. The in vitro model that was used to determine the heart protection was a limitation in the present study. The exact underlying mechanisms of the protective effects of GBR on myocardial $I / R$ injury are still unknown. Thus, the cardioprotection of GBR requires further study to confirm the protective effects. Previous observations have initially raised the possibility that GBR administration demonstrated a positive association with risk factors of cardiovascular diseases and GBR due to its bioactive compounds. The study in honey's beneficial natural therapeutic effect on cardiovascular diseases has suggested that phenolic and flavonoid components potentially provided antioxidant and anti-platelet activation. ${ }^{41}$ A similar suggestion has been reported by Daskalova et al. They found that phenolic compounds in berry showed antioxidant properties, anti-atherogenic effects, and cardioprotective effects in aging rats. ${ }^{42} \mathrm{~A}$ meta-analysis of prospective cohort studies had revealed a positive correlation between higher consumption of flavonoids and lower risk of mortality in cardiovascular diseases both in men and women. ${ }^{43}$ In the aging rat, long-term treatment of flavonoids significantly declined fibrosis, encouraged citrate synthase activity, and maintained the mitochondrial membrane potential. ${ }^{44}$ The dietary consumption of flavonoids in atherosclerosis, which is performed by macrophages RAW264.7 cells. This study highlighted that consumed flavonoids acted on prohibiting LPS-induced production of nitric oxide (NO), interleukin-6 (IL-6), tumor necrosis factor- $\alpha$ (TNF- $\alpha$ ), interleukin- 1 beta (IL- $1 \beta)$, and gene expression.

Also, the results indicated the potential effect of flavonoid on extracellular signal-regulated kinases (ERK), c-Jun N-terminal kinases (JNK), p38, p65, IкB $\alpha$, I $\kappa K \alpha / \beta$ phosphorylation, and nuclear factor-kappa B (NF- $\kappa \mathrm{B})$ nuclear translocation. ${ }^{45}$ Moreover, it has been suggested that GABA has anti-hypertension, anti-diabetes, anticancer, antioxidant, anti-inflammation, antimicrobial, antiallergy, hepato-protection, reno-protection, and intestinal protection. ${ }^{46}$ A study in islet $\beta$-cells revealed that GABA potentially exhibited its antidiabetic effects by modulating PI3K/Akt-dependent growth and survival pathways. According to the PI3K/Akt pathway's activation, cellular membrane depolarization and calcium influx lead to cell growth and survival. ${ }^{47}$ A study of GABA administration has been considerably discarded hydroxyl radical and thiobarbituric acid-reactive substances in both free medium and cultured media. ${ }^{48}$ Subsequent studies documented that GABA protected pancreatic cells and human umbilical vein endothelial cells against $\mathrm{H}_{2} \mathrm{O}_{2}$-induced oxidative stress by reducing cell death and diminishing reactive oxygen species (ROS) production, and promoting antioxidant activity. ${ }^{49,50}$ These all given evidence support the pharmaceutical properties of GBR on I/R injury-induced cardiomyocyte cells.

Nevertheless, the critical mechanism of GBR has not been established. Together with our results in this study offered the potential mechanism of GBR, which is associated with the process of cell death, apoptosis, mitochondria 
membrane potential, and mitochondria respiration on simulated I/R cardiomyocytes. Therefore, these findings suggested that GBR offered cardioprotection against I/R injury was partly mediated through mitochondrial metabolic function. During I/R injury progression, the opening of mitochondria permeability transitional pore serves as a critical factor for triggering several signaling cascades and protein kinases of cardiac cell death. ${ }^{13}$ Therefore, the cardioprotection of GBR might be related to the regulation of cell signaling pathways of survival and apoptosis. The hypotheses become apparent with our findings' results, which demonstrated the cardioprotective effect of GBR via mediating on p38 MAPK, pro-apoptotic Bax and Bcl-2, and caspase-3 protein expression (Data have not shown). Our findings may explain the potential mechanism on the cardioprotective effect of GBR in cardiomyocytes against I/R injury.

\section{Conclusion}

In conclusion, this study provides novel evidence that GBR pretreatment effectively protects against $I / R$ injury in $\mathrm{H} 9 \mathrm{c} 2$ cardiomyocyte through attenuation of cell death and apoptosis and maintenance of mitochondrial function. The underlying therapeutic activities are possibly associated with its bio-functional compounds. However, further studies are needed regarding the underlying mechanism of the cardioprotective effects of GBR in simulated ischemic/reperfusion injury.

\section{Ethics Approval and Consent to Participate}

The study was approved by the Ethical Committee, Kasetsart University, Thailand (ACKU 04059).

\section{Acknowledgments}

The authors are grateful to Miss Pichsinee Boonchuay, Siriraj Medical Research Center, Faculty of Medicine Siriraj Hospital, Mahidol University for her excellent technical assistance. The authors are also thankful to the Faculty of Veterinary Medicine, Kasetsart University, for providing facilities for the study.

\section{Author Contributions}

All authors made substantial contributions to conception and design, acquisition of data, or analysis and interpretation of data; took part in drafting the article or revising it critically for important intellectual content; agreed to submit to the current journal; gave final approval of the version to be published; and agree to be accountable for all aspects of the work.

\section{Funding}

This research was funded by the National Research Council of Thailand for The Royal Golden Jubilee Ph.D. Program to KD [grant no. PHD/0142/2561].

\section{Disclosure}

Dr Soontaree Petchdee reports a patent 1,803,002,749 pending. The authors report no other conflicts of interest in this work.

\section{References}

1. Benjamin EJ, Muntner P, Alonso A, et al. Heart disease and stroke statistics-2019 update: a report from the American heart association. Circulation. 2019;139(10):e56-e528. doi:10.1161/ CIR.0000000000000659

2. Kalogeris T, Baines CP, Krenz M, Korthuis RJ. Cell biology of ischemia/reperfusion injury. Int Rev Cell Mol Biol. 2012;298:229-317. doi:10.1016/B978-0-12-394309-5.00006-7

3. Jennings RB, Reimer KA. The cell biology of acute myocardial ischemia. Аппи Rev Med. 1991;42:225-246. doi:10.1146/annurev. me.42.020191.001301

4. Jennings RB. Historical perspective on the pathology of myocardial ischemia/reperfusion injury. Circ Res. 2013;113(4):428-438. doi:10.1161/CIRCRESAHA.113.300987

5. Jennings RB, Reimer KA. Acute myocardial ischemia: effects of reperfusion with arterial blood. Artif Cells Blood Substit Immobil Biotechnol. 1994;22(2):253-278. doi:10.3109/10731199409117419

6. Ibanez B, Heusch G, Ovize M. Evolving therapies for myocardial ischemia/reperfusion injury. $J \mathrm{Am}$ Coll Cardiol. 2015;65 (14):1454-1471. doi:10.1016/j.jacc.2015.02.032

7. Chen JR, Wei J, Wang LY, et al. Cardioprotection against ischemia/ reperfusion injury by QiShenYiQi Pill(R) via ameliorate of multiple mitochondrial dysfunctions. Drug Des Devel Ther. 2015;9:3051-3066. doi:10.2147/DDDT.S82146

8. Wu MY, Yiang GT, Liao WT, et al. Current mechanistic concepts in ischemia and reperfusion injury. Cell Physiol Biochem. 2018;46 (4):1650-1667. doi:10.1159/000489241

9. Veloso CD, Belew GD, Ferreira LL, et al. A mitochondrial approach to cardiovascular risk and disease. Curr Pharm Des. 2019;25 (29):3175-3194. doi:10.2174/1389203720666190830163735

10. Carpi A, Menabo R, Kaludercic N, Pelicci P, Di Lisa F, Giorgio M. The cardioprotective effects elicited by p66(Shc) ablation demonstrate the crucial role of mitochondrial ROS formation in ischemia/ reperfusion injury. Biochim Biophys Acta. 2009;1787(7):774-780. doi:10.1016/j.bbabio.2009.04.001

11. Li X, Jia P, Huang Z, et al. Lycopene protects against myocardial ischemia-reperfusion injury by inhibiting mitochondrial permeability transition pore opening. Drug Des Devel Ther. 2019;13:2331-2342. doi:10.2147/DDDT.S194753

12. Hausenloy DJ, Yellon DM. Myocardial ischemia-reperfusion injury: a neglected therapeutic target. J Clin Invest. 2013;123(1):92-100. doi:10.1172/JCI62874

13. Li X, Liu M, Sun R, Zeng Y, Chen S, Zhang P. Protective approaches against myocardial ischemia reperfusion injury. Exp Ther Med. 2016;12(6):3823-3829. doi:10.3892/etm.2016.3877 
14. Guerrero-Orriach JL, Escalona Belmonte JJ, Ramirez Fernandez A, Ramirez Aliaga M, Rubio Navarro M, Cruz Manas J. Cardioprotection with halogenated gases: how does it occur? Drug Des Devel Ther. 2017;11:837-849. doi:10.2147/DDDT.S127916

15. Chung MK. Vitamins, supplements, herbal medicines, and arrhythmias. Cardiol Rev. 2004;12(2):73-84. doi:10.1097/01. crd.0000091839.22076.f4

16. Caceres PJ, Martinez-Villaluenga C, Amigo L, Frias J. Maximising the phytochemical content and antioxidant activity of Ecuadorian brown rice sprouts through optimal germination conditions. Food Chem. 2014;152:407-414. doi:10.1016/j.foodchem.2013.11.156

17. Walter M, Marchesan E, Massoni PFS, da Silva LP, Sartori GMS, Ferreira RB. Antioxidant properties of rice grains with light brown, red and black pericarp colors and the effect of processing. Food Res Int. 2013;50(2):698-703. doi:10.1016/j. foodres.2011.09.002

18. Rahman K. Garlic and aging: new insights into an old remedy. Ageing Res Rev. 2003;2(1):39-56. doi:10.1016/s1568-1637(02) 00049-1

19. Valli G, Giardina EG. Benefits, adverse effects and drug interactions of herbal therapies with cardiovascular effects. $J$ Am Coll Cardiol. 2002;39(7):1083-1095. doi:10.1016/s0735-1097(02)01749-7

20. Kris-Etherton PM, Hecker KD, Bonanome A, et al. Bioactive compounds in foods: their role in the prevention of cardiovascular disease and cancer. Am J Med. 2002;113(Suppl 9B):71S-88S. doi:10.1016/ s0002-9343(01)00995-0

21. Ling WH, Cheng QX, Ma J, Wang T. Red and black rice decrease atherosclerotic plaque formation and increase antioxidant status in rabbits. J Nutr. 2001;131(5):1421-1426. doi:10.1093/jn/131.5.1421

22. Cho DH, Lim ST. Germinated brown rice and its bio-functional compounds. Food Chem. 2016;196:259-271. doi:10.1016/j. foodchem.2015.09.025

23. Gong ES, Luo S, Li T, et al. Phytochemical profiles and antioxidant activity of processed brown rice products. Food Chem. 2017;232:67-78. doi:10.1016/j.foodchem.2017.03.148

24. Ohtsubo K, Suzuki K, Yasui Y, Kasumi T. Bio-functional components in the processed pre-germinated brown rice by a twin-screw extruder. J Food Compost Anal. 2005;18(4):303-316. doi:10.1016/j. jfca.2004.10.003

25. Tian S, Nakamura K, Kayahara H. Analysis of phenolic compounds in white rice, brown rice, and germinated brown rice. J Agric Food Chem. 2004;52(15):4808-4813. doi:10.1021/jf049446f

26. Wu F, Yang N, Toure A, Jin Z, Xu X. Germinated brown rice and its role in human health. Crit Rev Food Sci Nutr. 2013;53(5):451-463. doi:10.1080/10408398.2010.542259

27. Prompunt E, Sanit J, Barrère-Lemaire S, et al. The cardioprotective effects of secretory leukocyte protease inhibitor against myocardial ischemia/reperfusion injury. Exp Ther Med. 2018;15(6):5231-5242. doi: $10.3892 /$ etm.2018.6097

28. Petchdee S, Laosripaiboon W, Jarussophon N, Kumphune S. Cardioprotective effects of germinated brown rice extract against myocardial ischemia reperfusion injury. High Blood Press Cardiovasc Prev. 2020;27(3):251-258. doi:10.1007/s40292-020-00378-x.

29. Patil SB, Khan MK. Germinated brown rice as a value added rice product: a review. J Food Sci Technol. 2011;48(6):661-667. doi:10.1007/s13197-011-0232-4

30. Chavan JK, Kadam SS. Nutritional improvement of cereals by sprouting. Crit Rev Food Sci Nutr. 1989;28(5):401-437. doi:10.1080/10408398909527508

31. Petchdee S, Laosripaiboon W, Jarussophon N. Cardiac protection of germinated brown rice extract in rabbit model of chronic myocardial infarction. Transl Anim Sci. 2020;4(2). doi:10.1093/tas/txaa067

32. Oh S-H, Moon Y-J, Soh J-R, Cha Y-S. Effect of water extract of germinated brown rice on adiposity and obesity indices in mice fed a high fat diet. Prev Nutr Food Sci. 2005;10:251-256. doi:10.3746/ jfn.2005.10.3.251.
33. Deng GF, Xu XR, Zhang Y, Li D, Gan RY, Li HB. Phenolic compounds and bioactivities of pigmented rice. Crit Rev Food Sci Nutr. 2013;53(3):296-306. doi:10.1080/10408398.2010.529624

34. Roohinejad S, Omidizadeh A, Mirhosseini H, et al. Effect of hypocholesterolemic properties of brown rice varieties containing different gamma amino butyric acid (GABA) levels on Sprague-Dawley male rats. J Food Agric Environ. 2010. doi:10.1234/4.2009.2501

35. Miura D, Ito $\mathrm{Y}$, Mizukuchi A, Kise M, Aoto H, Yagasaki K. Hypocholesterolemic action of pre-germinated brown rice in hepatoma-bearing rats. Life Sci. 2006;79(3):259-264. doi:10.1016/j. lfs.2006.01.001

36. Brown L, Rosner B, Willett WW, Sacks FM. Cholesterol-lowering effects of dietary fiber: a meta-analysis. Am J Clin Nutr. 1999;69 (1):30-42. doi:10.1093/ajcn/69.1.30

37. Estruch R, Martinez-Gonzalez MA, Corella D, et al. Effects of dietary fibre intake on risk factors for cardiovascular disease in subjects at high risk. J Epidemiol Community Health. 2009;63(7):582-588. doi:10.1136/jech.2008.082214

38. Kendall CWC, Esfahani A, Jenkins DJA. The link between dietary fibre and human health. Food Hydrocoll. 2010;24(1):42-48. doi:10.1016/j.foodhyd.2009.08.002

39. Oh $\mathrm{CH}$, Oh $\mathrm{SH}$. Effects of germinated brown rice extracts with enhanced levels of GABA on cancer cell proliferation and apoptosis. $J$ Med Food. 2004;7(1):19-23. doi:10.1089/ 109662004322984653

40. Pohjoismaki JL, Goffart S. The role of mitochondria in cardiac development and protection. Free Radic Biol Med. 2017;106:345-354. doi:10.1016/j.freeradbiomed.2017.02.032

41. Olas B. Honey and its phenolic compounds as an effective natural medicine for cardiovascular diseases in humans? Nutrients. 2020;12 (2). doi:10.3390/nu12020283.

42. Daskalova E, Delchev S, Peeva Y, et al. Antiatherogenic and cardioprotective effects of black chokeberry (aronia melanocarpa) juice in aging rats. Evid Based Complement Alternat Med. 2015;2015:717439. doi:10.1155/2015/717439.

43. Kim Y, Je Y. Flavonoid intake and mortality from cardiovascular disease and all causes: a meta-analysis of prospective cohort studies. Clin Nutr ESPEN. 2017;20:68-77. doi:10.1016/j.clnesp.2017.03.004

44. Testai L, Piragine E, Piano I, et al. The citrus flavonoid naringenin protects the myocardium from ageing-dependent dysfunction: potential role of SIRT1. Oxid Med Cell Longev. 2020;2020:4650207. doi:10.1155/2020/4650207

45. Shen C-Y, Lin -J-J, Jiang J-G, Wang T-X, Zhu W. Potential roles of dietary flavonoids from Citrus aurantium L. var. amara Engl. in atherosclerosis development. Food Funct. 2020;11(1):561-571. doi:10.1039/C9FO02336D

46. Ngo DH, Vo TS. An updated review on pharmaceutical properties of gamma-aminobutyric acid. Molecules. 2019;24(15). doi:10.3390/ molecules 24152678

47. Soltani N, Qiu H, Aleksic M, et al. GABA exerts protective and regenerative effects on islet beta cells and reverses diabetes. Proc Natl Acad Sci U S A. 2011;108(28):11692-11697. doi:10.1073/ pnas. 1102715108

48. Md Zamri ND, Imam MU, Abd Ghafar SA, Ismail M. Antioxidative effects of germinated brown rice-derived extracts on $\mathrm{H} 2 \mathrm{O} 2$-induced oxidative stress in HepG2 cells. Evid Based Complement Alternat Med. 2014;2014:371907. doi:10.1155/2014/371907.

49. Tang X, Yu R, Zhou Q, Jiang S, Le G. Protective effects of gamma-aminobutyric acid against $\mathrm{H} 2 \mathrm{O} 2$-induced oxidative stress in RIN-m5F pancreatic cells. Nutr Metab. 2018;15:60. doi:10.1186/ s12986-018-0299-2

50. Zhu Z, Shi Z, Xie C, Gong W, Hu Z, Peng Y. A novel mechanism of gamma-aminobutyric acid (GABA) protecting human umbilical vein endothelial cells (HUVECs) against $\mathrm{H} 2 \mathrm{O} 2$-induced oxidative injury. Comparative biochemistry and physiology. Toxicol Pharmacol. 2019;217:68-75. doi:10.1016/j.cbpc.2018.11.018. 


\section{Publish your work in this journal}

Drug Design, Development and Therapy is an international, peerreviewed open-access journal that spans the spectrum of drug design and development through to clinical applications. Clinical outcomes, patient safety, and programs for the development and effective, safe, and sustained use of medicines are a feature of the journal, which has also been accepted for indexing on PubMed Central. The manuscript management system is completely online and includes a very quick and fair peer-review system, which is all easy to use. Visit http://www. dovepress.com/testimonials.php to read real quotes from published authors. 